\title{
Hydromagnetic Blood Flow through a Uniform Channel with Permeable Walls Covered by Porous Media of Finite Thickness
}

\author{
K. Ramakrishnan ${ }^{1 \dagger}$ and K. Shailendhra ${ }^{2}$ \\ ${ }^{1}$ Dept. of Math, Sri Krishna College of Eng. and Technology, Sugunapuram, Kuniamuthur, Coimbatore, Tamilnadu, \\ 641008, India \\ ${ }^{2}$ Dept. of Math, Amirta School of Eng., Amirta Vishwa Vidyapeetham, Ettimadai,Coimbatore, Tamilnadu, 641 105, \\ India \\ †Corresponding Author Email: murali_19722002@yahoo.co.in
}

(Received September 25, 2010; accepted October 9, 2011)

\begin{abstract}
The effects of Hartmann number, porous parameter and Darcy velocity on the steady flow of a viscous incompressible slightly conducting fluid through a uniform channel bounded by porous media of finite thickness under a uniform ransverse magnetic field are considered. It is assumed that the thickness of the porous media is much smaller than the width of the flow channel as in the case of blood flow in arteries and accordingly the BJR slip boundary condition has been employed. The effects of all the above parameters on the axial velocity of the flow and the shear stress have been investigated. Finally, these results are compared with a earlier problem of MHD flow through a uniform channel covered by porous media of infinite thickness where the BJ slip boundary condition has been employed.
\end{abstract}

Keywords: BJR slip condition, Blood flow, MHD, Porous media, Shears stress.

\section{NOMENCLATURE}

$\begin{array}{ll}\rho & \text { density of the fluid } \\ \mu & \text { viscosity of the fluid } \\ \sigma_{e} & \text { electrical conductivity } \\ B_{0} & \text { applied magnetic field in the y direction } \\ H & \text { thickness of the porous layer } \\ h & \text { thickness of the fluid layer } \\ Q_{1} & \text { Darcy velocity }\end{array}$

\section{INTRODUCTION}

Biomagnetic fluid dynamics (BFD) is a relatively new area in fluid mechanics investigating the fluid dynamics of biological fluids in the presence of magnetic field. A biomagnetic fluid is a fluid that exists in a living creature and its flow is influenced by the presence of a magnetic field. The most characteristic biomagnetic fluid is blood, which behaves as a magnetic fluid, as suggested by Higashi et al. (1993) due to the complex interaction of the intercellular protein. The effects of Lorentz force on the flow of blood in the presence of a stenosis are (i) to reduce the high shear stress caused by stenosis and hence to prevent the damage to the red and endothelial cells and (ii) to delay the transition from laminar to turbulent flow (Chandrasekhar 1961; Rudraiah 1962) inside the blood vessel and thus

$\begin{array}{ll}\lambda & \text { viscosity factor } \\ n_{f} & \text { the net flux through the channel } \\ u_{b 1}, u_{b 2} & \text { slip velocities } \\ k & \text { permeability of the porous material } \\ \sigma_{p} & \text { porous parameter } \\ \alpha & \text { slip parameter } \\ M & \text { Hartmann number }\end{array}$

reducing high intensity shear zones, which are unfavorable to the blood and arterial wall. During the last few decades, the flow of biological fluids under the influence of a magnetic field has been taken up by many researchers due to numerous possible applications in the field of bioengineering and medicine (Haik 1990; Ruuge 1993; Misra and Shit 2007).

Many investigators (Chaturani and Kaloni 1976; Chaturani and Upadhya 1979; Shukla et al. 1980; Chaturani and Biswas 1983; Majhi and Usha 1984; Philip and Peeyush Chandra 1996) have theoretically studied the flow of blood through uniform and stenosed tubes and analyzed the influence of slip velocity or peripheral plasma layer thickness on the flow variables such as velocity and wall shear stress. Recently, mathematical model of biomagnetic fluid dynamics, 
suitable for the description of the Newtonian blood flow under the action of an applied magnetic field, is proposed by Tzirtzilakis (2005).

MHD flow through a channel with rigid boundaries are not of much use in understanding the characteristics of flow in arteries, because they are bounded by tissues which are idealized into a porous medium where one has to use a slip condition at the binding surface. It is important for the study of motion of contact line where the effective slip of the interface reverses the singularity in the rate of strain which was otherwise introduced by the no-slip condition. In the present situation the interface may be a smooth surface or a rough surface depending upon the solid matrix of the porous material. In the former case the surface can be considered as a nominal surface postulated by Beaver and Joseph (1967) called as BJ slip condition) at which BJ slip condition exists due to transfer of momentum from fluid to fluid saturated porous media.

The hydromagnetic steady flow of a viscous conducting fluid in a channel with slip at the permeable boundaries has been investigated by Makinde and Osalusi (2006). Most recently, Ramakrishnan and Shailendhra (2011) analyzed the combined effects of Hartmann number and porous parameter on the steady flow in a channel of uniform width covered by porous media using BJ-slip condition.

The BJ-slip condition is independent of the thickness of the porous layer and hence valid when the thickness of the porous layer is very much larger than the thickness of the fluid layer. In many industrial and biochemical applications the thickness of the porous layer is comparable to that of fluid layer and hence the slip condition should involve the thickness of the layer. Rudraiah (1985) has derived the slip condition known as BJR slip condition involving the thickness of the layer. This BJR slip condition reduces to BJ condition for large thickness of the layer. Rudraiah et al. (2006) investigated the electrohydrodynamic dispersion of macromolecular components in a biological bearing using BJ and BJR slip conditions. Rudraiah et al. (2007) studied the electrohydro- dynamic dispersion of macromolecular components in a biological bearing consisting of a poorly conducting synovial fluid both in the cavity of the bones and in the bounding porous cartilage of finite thickness using BJR slip condition. Electrohydro-dynamic dispersion due to pulsatile flow in a channel bounded by porous layer of smart material was studied by $\mathrm{Ng}$ et al. (2008) using both BJ and BJR slip conditions.

A study on the effect of electromagnetic field on the flow in a channel with surface bounded by porous media of finite thickness using BJR slip condition is of great use in understanding and prevention of arterial diseases. So far, no attempt has been made to investigate the effect of magnetic field on the blood flow through uniform channel bounded by porous media with finite thickness using BJR slip condition.

\section{Formulation OF THE Problem}

Here, steady, laminar, highly viscous, conducting, hydromagnetic blood flow through a straight channel with permeable walls covered by porous media of finite thickness is considered. It is well known that at high shear-rates blood behaves like a Newtonian fluid during flows through large blood vessels (Shivakumar et al. 1986; Misra et al. 1986; Copley 1990 and Tzirtzilakis 2005). But in particular situations blood may behave as a non-Newtonian fluid, even in large arteries. It is also worthwhile to mention here that although blood is nonNewtonian suspension of cells in plasma, MacDonald (1974) remarked that for vessels of radius greater than $0.025 \mathrm{~cm}$, blood may be considered as a homogeneous Newtonian fluid. Here we assume that blood behaves like a homogeneous conducting Newtonian fluid, with constant density $\rho$, viscosity $\mu$ and electrical conductivity $\sigma_{\mathrm{e}}$. Further, it is well known that blood flow in arteries are pulsatile Burton (1966). However, we have considered the flow here to be steady with an understanding that all physical quantities like velocity, pressure etc are representative time averages over one period.

It is further assumed that the channel is symmetrical to the $x$-axis. The porous layer is assumed to be homogeneous, isotropic and densely packed so that the usual Darcy law is valid. A uniform magnetic field $B_{0}$ is applied in the y direction. We note that, as stated earlier, the BJ-slip condition is valid for large thickness of the porous layer $(H)$ compared to thickness of the fluid layer $(h)$, i.e. $H>h$. In many practical cases, particularly in biomechanical problems, the thickness of the porous layer is usually small, compared to the thickness of fluid layer (i.e. $H \leqslant h$ ). Besides, the presence of a thin boundary layer in the porous region of Brinkmann type has to be taken into account. Considering these factors, the BJR slip condition proposed by Rudraiah (1985) is taken as boundary condition to solve the equations of motion.

Consider a cartesian coordinate system $(x, y)$ where $x$ lies along the center of the channel, $y$ is the distance measured in the normal section such that $y=h$ is the channel's half width. Let $u$ and $v$ be the velocity components in the directions of $x$ and $y$ increasing respectively (Refer Fig.1).

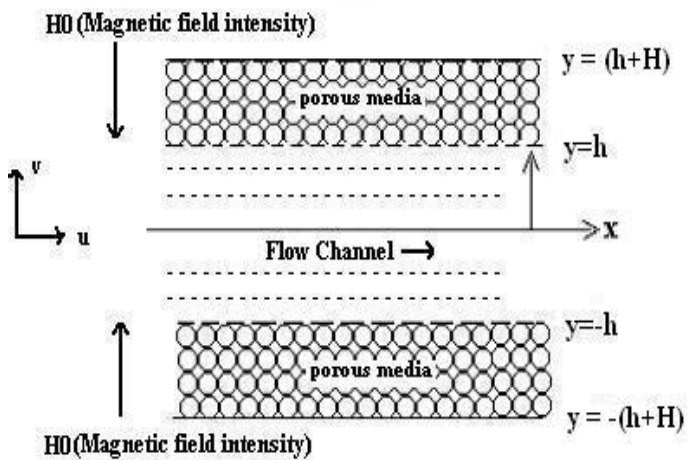

Fig. 1. Physical Configuration

Then, the continuity and Navier-Stokes equations governing the flow are:

$\frac{\partial u}{\partial x}+\frac{\partial v}{\partial y}=0$ 
$u \frac{\partial u}{\partial x}+v \frac{\partial u}{\partial y}=-\frac{1}{\rho} \frac{\partial \rho}{\partial x}+v \nabla^{2} u-\frac{\sigma_{e} B_{0}^{2} u}{\rho}$

$u \frac{\partial v}{\partial x}+v \frac{\partial v}{\partial y}=-\frac{1}{\rho} \frac{\partial \rho}{\partial y}+v \nabla^{2} v$

where

$\nabla^{2}=\frac{\partial^{2}}{\partial x^{2}}+\frac{\partial^{2}}{\partial y^{2}}$

$B_{0}$ is the applied magnetic field in the $y$-direction, and $p$ the pressure.

It is to be noted that when the characteristic length of the flow is small the magnetic Reynolds number cannot exceed unity unless the flow is turbulent Roberts(1967). The equations of motions, neglecting inertia (for we are considering highly viscous fluid, Shivakumar et al. (1986) and induced magnetic field (the fluid is of small electrical conductivity with magnetic Reynolds number much less than unity so that the induced magnetic field can be neglected in comparison with the applied magnetic field) are,

$\frac{\partial^{2}}{\partial y^{2}}-\frac{\sigma_{e} B_{0}^{2}}{\mu} u=\frac{1}{\mu} \frac{\partial \rho}{\partial x}$

$\frac{1}{\mu} \frac{\partial \rho}{\partial y}=0$

$v=0$

and the velocity distribution in the porous layer is given by the Darcy's law,

$Q_{x}=-\frac{k}{\mu} \frac{\partial \rho}{\partial x}$ and $Q_{y}=-\frac{k}{\mu} \frac{\partial \rho}{\partial y}$

These equations are solved using the BJR slip boundary conditions (Rudraiah (1985)) given by,

$\frac{\partial u}{\partial y}=\delta_{1} Q_{1}+\delta_{2}\left(u_{b 1}-Q_{1}\right) \quad$ at $y=-h$

$\frac{\partial u}{\partial y}=-\delta_{1} Q_{1}-\delta_{2}\left(u_{b 2}-Q_{1}\right) \quad$ at $\quad y=h$

$v=\varepsilon Q_{y}, \quad Q_{1}=-\frac{k}{\mu} \frac{\partial \rho}{\partial x}$

$\int_{-h}^{h} u(y) d y=n f$

Where

$\delta_{1}=\frac{\lambda \sqrt{\lambda} \sigma_{p} \varepsilon}{\delta_{0} \sinh \left(\frac{\sigma_{p}}{\sqrt{\lambda}}\right)}, \delta_{1}=\frac{\sqrt{\lambda}}{\delta_{0}} \sigma_{p} \operatorname{coth}\left(\frac{\sigma_{p}}{\sqrt{\lambda}}\right)$

$\sigma_{p}=\frac{H}{\sqrt{k}} \sigma_{p}, \delta_{0}=\frac{H}{h}$ and $u_{b 1}=u_{b 2}=u_{b}$

Here, $\lambda$ is a positive constant called viscosity factor, $\mathrm{nf}$ is the net flux through the channel, $u_{b 1}$ and $u_{b 2}$ are the slip velocities, $Q_{l}$ is the Darcy velocity, $k$ is the permeability of the porous material and $\sigma_{\mathrm{p}}$ is called the porous parameter.

\section{Solution of The Problem}

In the case of a uniform channel, the wall slope is everywhere negligible and the components of velocity and pressure gradient are approximately equal. After eliminating the pressure term between (4) and (5), the non dimensional basic equations are attained as

$\frac{\partial^{3} u}{\partial y^{3}}+M^{2} \frac{\partial u}{\partial y}=0$

since $\mathrm{u}$ is a function of $\mathrm{y}$ alone, where

$y^{*}=\frac{y}{h}$ and $M^{2}=\frac{B_{0}^{2} h^{2} \sigma_{e}}{\mu}$

is the square of the Hartmann number and $*$ is dropped for simplicity.

The corresponding boundary conditions become,

$\frac{d u}{d y}=\delta_{1} Q_{1}+\delta_{2}\left(u_{b 1}-Q_{1}\right)$ at $y=-1$

$\frac{d u}{d y}=-\delta_{1} Q_{1}-\delta_{2}\left(u_{b 2}-Q_{1}\right)$ at $y=1$

$v=\varepsilon Q_{y}, \quad Q_{1}=-\frac{k}{\mu} \frac{\partial p}{\partial x}$

$\int_{-1}^{1} u(y) d y=\frac{n_{f}}{h}$

Solving Eq. (14) subject to the conditions (16) to (19) we have,

$u(y)=\frac{n_{f}}{2 h}+\frac{\chi}{2 h}\left[\frac{2 h Q_{1}\left(\delta_{1}-\delta_{2}\right)+\delta_{2} n_{f}}{\left(M^{2}-\delta_{2}\right) \sinh (M)+\delta_{2} M \cosh (M)}\right]$

where

$n_{f}=\frac{2 h}{\sigma_{p}^{2} M^{2}}\left[\frac{\left(\chi_{1}+\chi_{2}\right)\left(-\frac{1 \partial p}{u_{f} \partial x}\right)}{M^{2} \sinh (M)+\delta_{2} M \cosh (M)}\right]$

$\chi=\sinh (M)-M \cosh \left(M_{y}\right)$

$\chi_{1}=\left[h^{2} \sigma_{p}^{2}\left(M^{2}-\delta_{2}\right)+H^{2}\left(\delta_{2}-\delta_{1}\right) M^{2}\right] \sinh (M)$

$\chi_{2}=h^{2} \sigma_{p}^{2} M \delta_{2} \cosh (M)$

and the slip velocity is given by,

$u_{b}=\frac{1}{2 h}\left[\frac{\beta_{1}+\beta_{2}}{M^{2} \sinh (M)+\delta_{2} \zeta}\right]$

where

$\zeta=\sinh (M)-M \cosh \left(M_{y}\right)$

$\beta_{1}=n_{f} M^{2} \sinh (M)$

$\beta_{2}=2 h Q_{1}\left(\delta_{1}-\delta_{2}\right) \zeta$

We note that when thickness of the porous media $(H)$ is infinity, the BJR slip condition coincides with the BJ slip condition Refer Rudraiah (1985). In other words, the slip parameter $(\alpha)$ used by Beavers-Joseph (1967) has the physical meaning of the viscosity factor $(\lambda)$.

When $H \rightarrow \infty$, the axial velocity is given by

$u(y)=\frac{n_{f}}{2 h}+\frac{\sigma M}{2 h}\left[\frac{\left(n_{f}-2 h Q_{1}\right) \chi}{\left(M^{2}-\alpha \sigma\right) \sinh (M)+\alpha \sigma M \cosh (M)}\right]$

where

$n_{f}=\frac{2 h^{3}}{\sigma M^{2}}\left[\frac{\Psi_{1}+\Psi_{2}}{M^{2} \sinh (M)+\alpha \sigma M \cosh (M)}\right]\left(-\frac{1}{\mu} \frac{\partial p}{\partial x}\right)$

$\chi=\sinh (M)-M \cosh \left(M_{y}\right)$

$\Psi_{1}=\left(\alpha M^{2}+\sigma M^{2}-\alpha \sigma^{2}\right) \sinh (M)$

$\Psi_{2}=\alpha \sigma^{2} M \cosh (M)$

and the slip velocity is, 
$u_{B}=\frac{1}{2 h}\left[\frac{n_{f} M^{2} \sinh (M)-2 \alpha \sigma h Q_{1} \xi}{M^{2} \sinh (M)-\sigma h \xi}\right]$

where

$\xi=\sinh (M)-M \cosh (M)$

Equations (23) to (25) are already reported in our earlier work Ramakrishnan and Shailendhra (2011) and are provided here only for the sake of comparison.

\section{RESULTS AND DISCUSSION}

Since we are dealing with the problem of blood flow through a uniform channel bounded by porous media, we fix the values for the parameters as follows: $\mu=$ $0.004 \mathrm{kgm}^{-1} \mathrm{~s}^{-1}, h=0.002 \mathrm{~m}$ Tzirtzilakis (2005) and $H=$ $0.001 \mathrm{~m}$. Also, we vary the values of the Hartmann number as : $\mathrm{M}=0,2,3.5,5$ Chandrasekhara et al. (1980) and the porous parameter $\sigma_{\mathrm{p}}=10^{1}, 10^{2}, 10^{3}$ Shivakumar et al. (1986). The effects of the various parameters on the axial velocity are discussed with the help of graphs and those on the slip velocity and shear stress are done with the help of tables.

From Fig.2 and Fig.3, it is clear that, in the absence as well as presence of magnetic field, the effect of the porous parameter on the axial velocity is to reduce it. However, it is seen that for higher values of the Hartmann number $(M \geq 3.5)$, the flow is reversed.

When Fig. 2 and Fig.3 are compared with Fig.4 and Fig.5, it is seen that when the magnetic field is absent or when it is of low intensity, the effect of porous parameter $\left(\sigma_{p}\right)$ on the axial flow velocity is to decrease it. The retardation of the flow by porous parameter is felt only in the central core region and not near the walls.

Fig.6 and Fig.7 depict the fact that the effect of magnetic field on the axial velocity is to reduce it, for all possible values considered for the porous parameter $\left(\sigma_{p}\right)$. However when the porous parameter is increased from $10^{2}$, there is no significant difference in the axial velocity, since in this case the walls behave like impermeable walls.

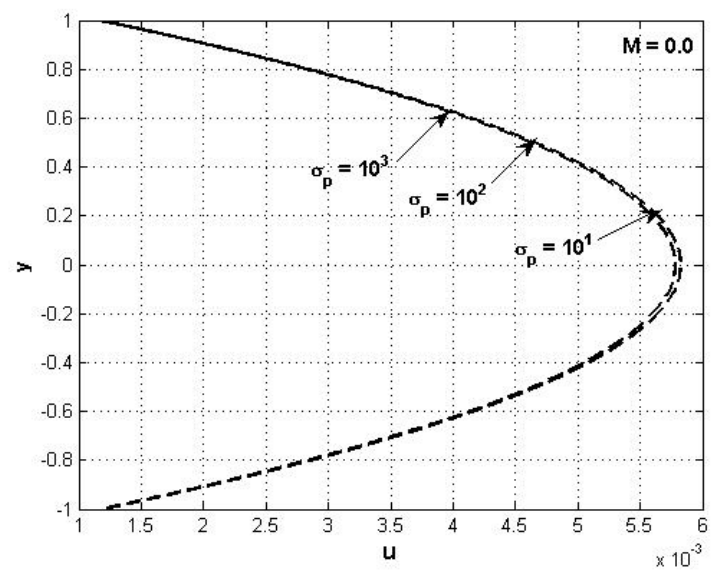

Fig. 2. Axial velocity for $M=0.0$ and different values of $\sigma p$

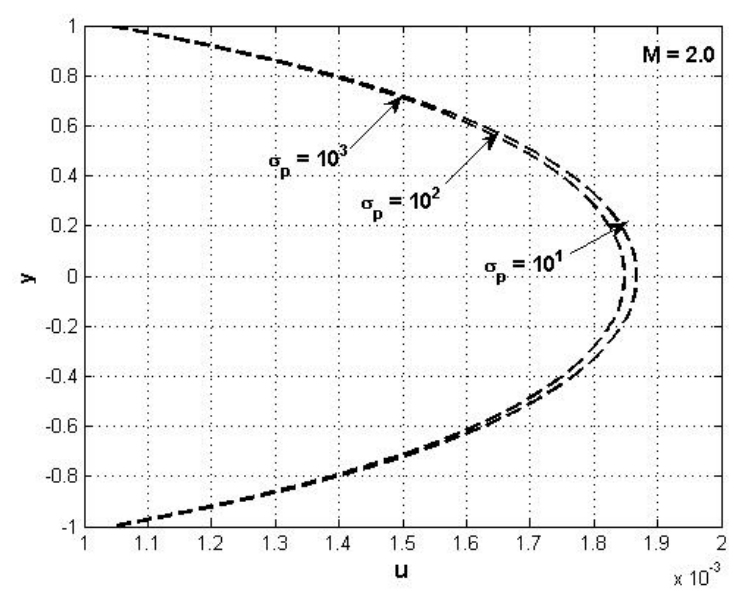

Fig. 3. Axial velocity for $M=2.0$ and different values of $\sigma p$

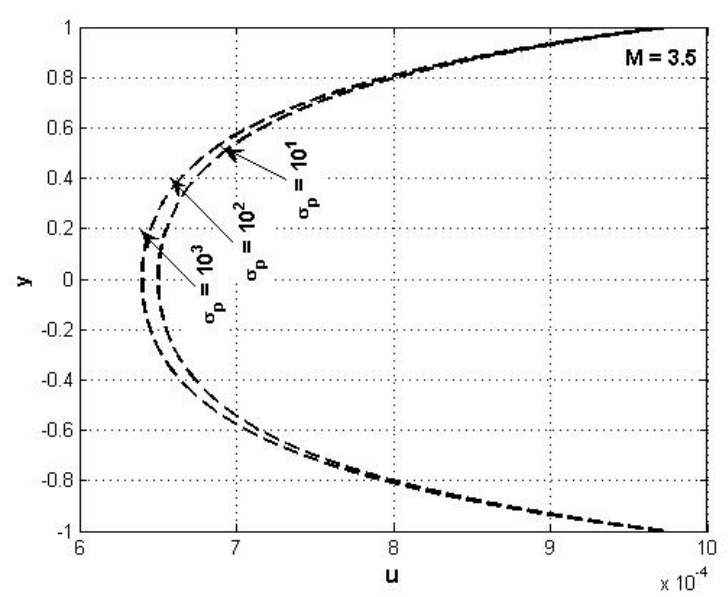

Fig. 4. Axial velocity for $M=3.5$ and different values of $\sigma p$

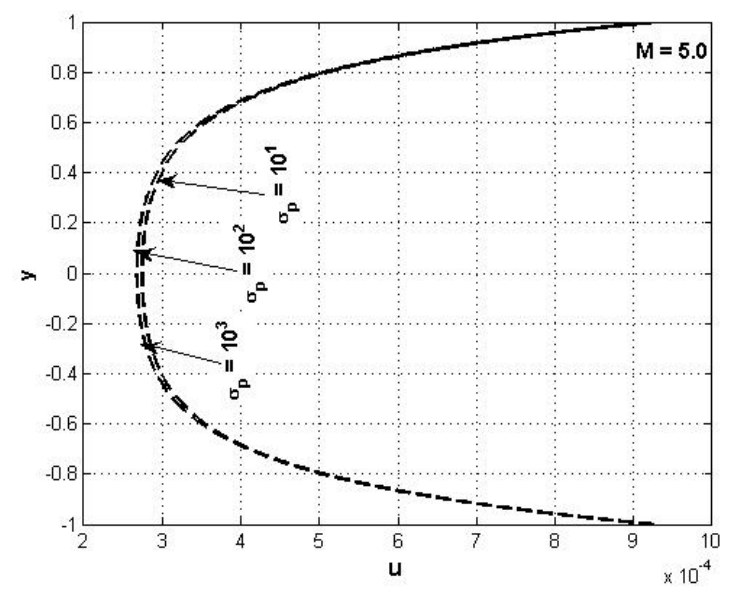

Fig. 5. Axial velocity for $M=5.0$ and different values of $\sigma p$ 


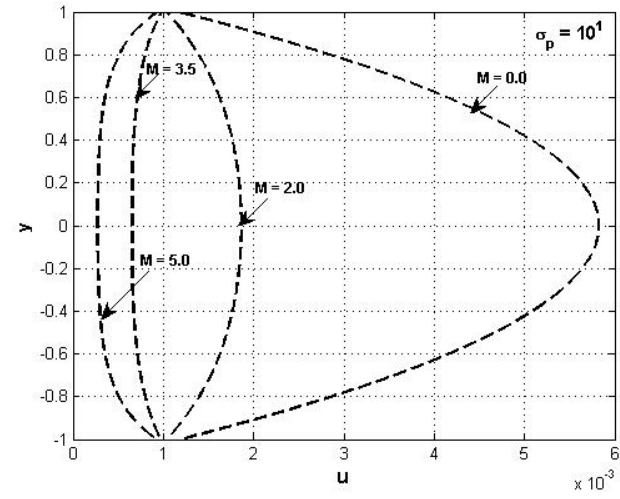

Fig. 6. Axial velocity for $\sigma p=10^{1}$ and different values of $\mathrm{M}$

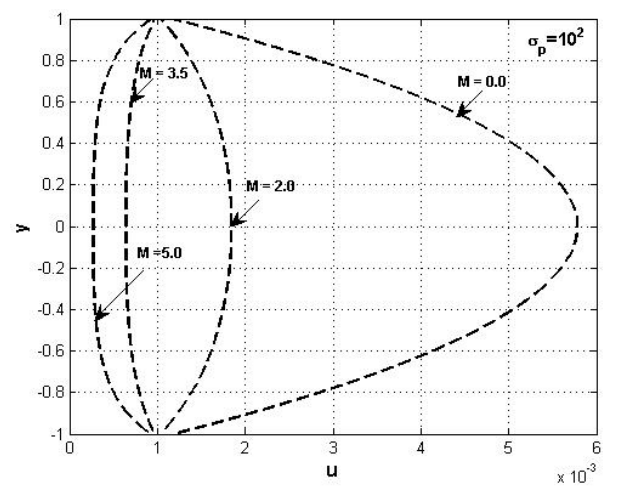

Fig. 7. Axial velocity for $\sigma p=10^{2}$ and different values of $\mathrm{M}$

In many biological processes the thickness of the porous layer is comparable to that of fluid layer.However in a few earlier references, (Chandrasekhara et al. 1980; Shivakumar et al. 1986; Mankinde and Osalusi 2006) the authors had analyzed blood flow in the case of straight channel covered by porous media with infinite thickness, by stating that, the results of the problem may also be useful to improved understanding and management of human health. Hence, the results of our present study involving porous media of finite thickness (BJR condition) have been compared with those of earlier works with porous media of infinite thickness (BJ condition), especially Ramakrishnan and Shailendhra (2011). Indeed, we make such a comparison through Figs. 8 to 14 and in these figures the curves with dotted lines pertain to BJ slip conditions and these are in excellent agreement with our earlier work (2011).

From Figs. 8 to 11 , it is observed that axial velocity decreases with increasing porous parameter $\left(\sigma_{p}\right)$ for a fixed value of Hartmann number $(M)$ in both infinite and finite thickness porous media. In the case of porous media of finite thickness using BJR slip conditions, the flow is found to be slower than the corresponding flow for the porous media of infinite thickness (using BJ condition) for small values of porous parameter upto $10^{1}$. For higher values of the porous parameter $\left(10^{2}\right.$ and above) the flow with BJR conditions is found to be faster than the corresponding flow with $\mathrm{BJ}$ conditions (Figs. 8, 9, 10).

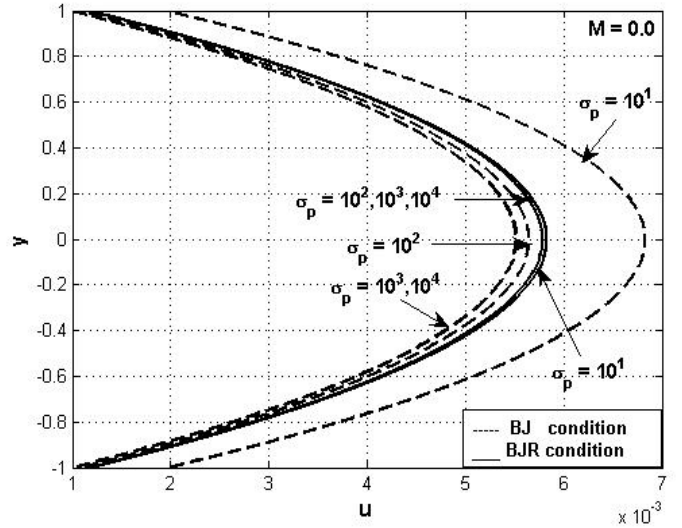

Fig. 8. Comparison of axial velocity for $M=0.0$ and different values of $\sigma p$ using BJ and BJR slip conditions

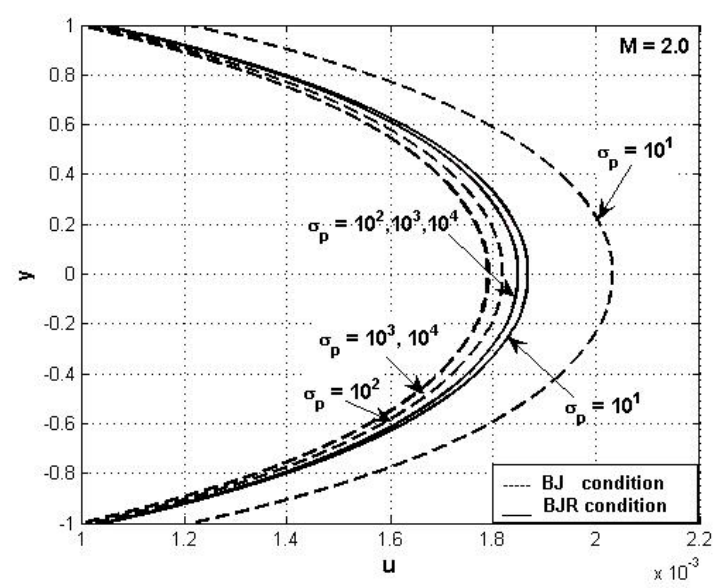

Fig. 9. Comparison of axial velocity for $\mathrm{M}=2.0$ and different values of $\sigma p$ using BJ and BJR slip conditions

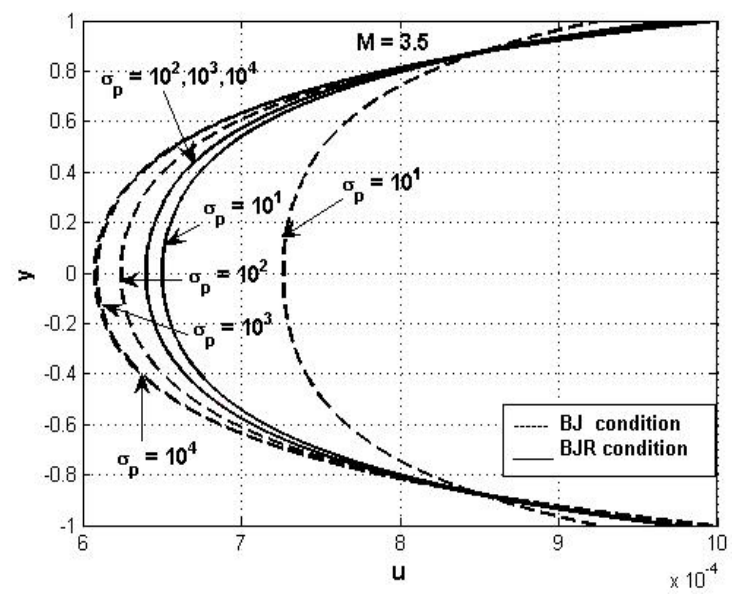

Fig. 10. Comparison of axial velocity for $M=3.5$ and different values of $\sigma p$ using BJ and BJR slip conditions 


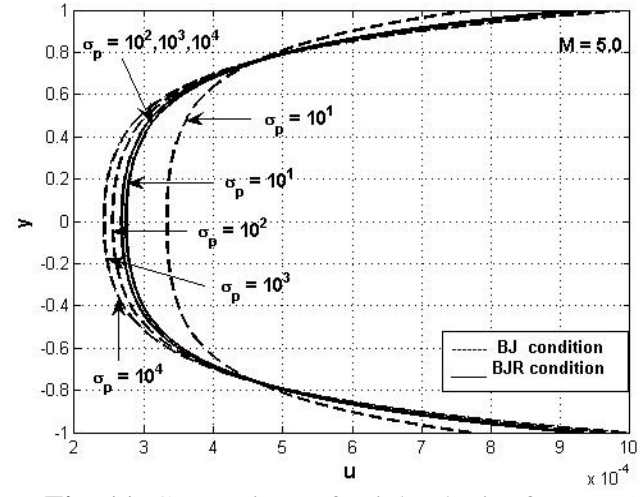

Fig. 11. Comparison of axial velocity for $M=5.0$ and different values of $\sigma p$ using BJ and BJR slip conditions

From Figs. $12-14$, it is clear that the axial velocity is decreased with increasing of the Hartmann number in both infinite (using BJ condition) and finite (using BJR slip condition) thickness porous media. In particular from Fig. 12, it is noted that for $\sigma_{p}=10^{l}$ and in the absence of magnetic field i.e., $M=0.0$, the axial velocity is significantly higher in the case of infinite thickness porous media using BJ slip condition than in the case of finite thickness porous media using BJR slip condition. From Fig. 13 - 14, it is observed that the axial velocity has higher values in the case of BJR slip condition than in the case of $\mathrm{BJ}$ slip condition for $\sigma_{p}=$ $10^{2}$ and $10^{3}$.

Tables 1 and 2 depict the distribution of slip velocity $\left(u_{b}\right)$ for two different thickness of the porous medium. In all the cases, the slip velocity can be seen to decrease for increasing Hartmann number $(M)$ and the porous parameter $\left(\sigma_{p}\right)$. It is also observed that from $\sigma_{\mathrm{p}}=10^{8}$ (not shown in the table), the slip velocity distribution is constant because walls behave like impermeable rigid walls in this case. Comparing Tables 1 and 2, it is noted that if we increase the thickness of the porous media, for all possible values of Hartmann number, the slip velocity decreases when porous parameter is increased from 10 to $10^{3}$. But when we increase the value of porous parameter from $10^{4}$, the slip velocity starts increasing.

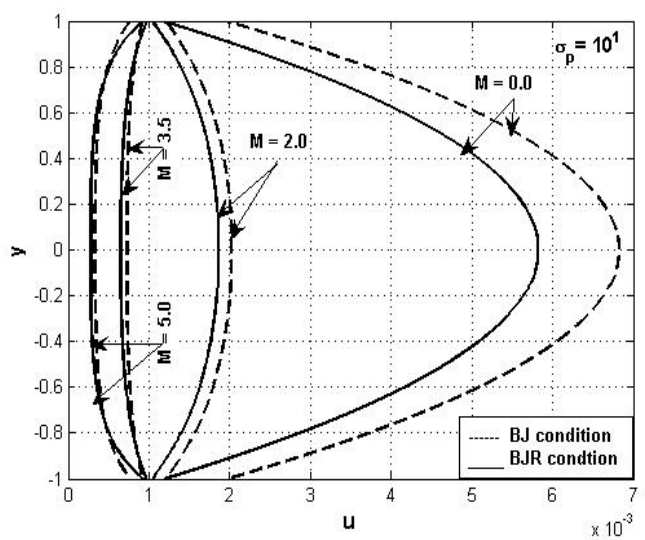

Fig. 12. Comparison of axial velocity for $\sigma p=10^{1}$ and different values of $\mathrm{M}$ using BJ and BJR slip conditions

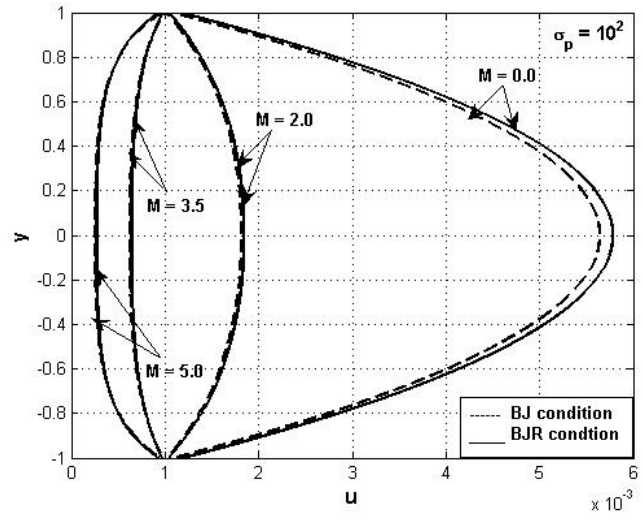

Fig. 13. Comparison of axial velocity for $\sigma p=10^{2}$ and different values of $\mathrm{M}$ using BJ and BJR slip conditions

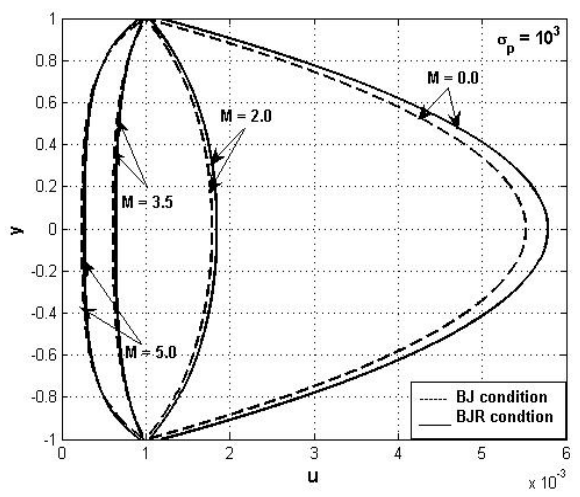

Fig. 14. Comparison of axial velocity for $\sigma p=10^{3}$ and different values of $\mathrm{M}$ using $\mathrm{BJ}$ and BJR slip conditions

The wall shear stress at the lower wall is given by

$\tau_{x y}=-\frac{M^{2}}{2 h^{2}}\left[\frac{\eta}{\left(M^{2}-\delta_{2}\right) \sinh (M)+M \delta_{2} \cosh (M)}\right]$

where

$\eta=2 h Q_{1}\left(\delta_{1}-\delta_{2}\right) \sin h(M)+\delta_{2} n_{f} \sinh (M)$

When $H \rightarrow \infty$, the wall shear stress in the lower wall in the case of $\mathrm{BJ}$ slip condition is given by

$\tau_{x y}=\frac{\bar{\alpha} \sigma}{2 h} \frac{M^{2}}{h}\left[\frac{\left[n_{f}-2 h Q_{1}\right] \sinh (M)}{\left(M^{2}-\bar{\alpha} \sigma\right) \sinh (M)+\bar{\alpha} \sigma M \cosh (M)}\right]$

This result has been already reported in our earlier work (2011).

The effect of the porous parameter and the magnetic field on the shear stress at the lower wall $y=-h$ is presented from Tables 4 to 6 . It should be noted that Table 6 is already presented in our earlier work (2011) and it is presented here only for the sake of comparison.

From Tables 3 to 5 , it is evident that the effect of magnetic field is to reduce the shear stress for all possible values of the porous parameter under consideration. The effect of the porous parameter, both in the absence and the presence of magnetic field is to increase the shear stress. But, when the porous parameter $\sigma_{\mathrm{p}}$ is increased beyond $10^{7}$ (not shown in the table) for different thickness of the porous media, the effect of the porous parameter on shear stress is 
insignificant, since the walls behave like impermeable rigid walls.

It is obvious from Tables 4 to 6 that, the effect of magnetic field is to decrease the shear stress numerically, when the porous parameter $\sigma_{\mathrm{p}}$ is increased from $10^{1}$ to $10^{2}$. However, when $\sigma_{\mathrm{p}}$ is increased beyond $10^{3}$, it is seen that the shear stress remains positive indicating the fact that the stress acts in the upward direction. This is possibly due to the flow reversal observed earlier.
Finally, it was observed that the values of shear stress at the upper wall are same in magnitude as that on the lower wall except that the sign is changed, which in turn indicates the fact that shear stress of equal magnitude act on both the walls but in opposite directions.

From Tables 5 and 6, it is observed that the shear stress at the walls for the flow with finite thickness porous media (BJR condition) is greater than the corresponding shear stress for the flow with infinite thickness porous media (BJ condition). This shows that studies with BJ conditions under estimate the shear stress.

Table 1 Slip velocity distribution for different values of porous parameter $\left(\sigma_{p}\right)$ and Hartmann number $(M)(H=h / 4)$

\begin{tabular}{|c|c|c|c|c|c|}
\hline$M \downarrow \quad \sigma_{0} \rightarrow$ & $10^{1}$ & $10^{2}$ & $10^{3}$ & $10^{4}$ & $10^{5}$ \\
\hline 0.0 & 0.0972 & 0.0098 & 0.00110 & 0.00021 & 0.00013 \\
\hline 2.0 & 0.0964 & 0.0097 & 0.00100 & 0.00015 & 0.00006 \\
\hline 3.5 & 0.0953 & 0.0096 & 0.00098 & 0.00012 & 0.00004 \\
\hline 5 & 0.0940 & 0.0094 & 0.00096 & 0.00011 & 0.00003 \\
\hline
\end{tabular}

Table 2 Slip velocity distribution for different values of porous parameter $\left(\sigma_{p}\right)$ and Hartmann number $(M)(H=3 h / 4)$

\begin{tabular}{|c|c|c|c|c|c|}
\hline$M \downarrow \quad \sigma_{0} \rightarrow$ & $10^{1}$ & $10^{2}$ & $10^{3}$ & $10^{4}$ & $10^{5}$ \\
\hline 0.0 & 0.0920 & 0.0095 & 0.00130 & 0.00045 & 0.00037 \\
\hline 2.0 & 0.0899 & 0.009 & 0.00110 & 0.00025 & 0.00017 \\
\hline 3.5 & 0.0871 & 0.0088 & 0.00096 & 0.00018 & 0.00010 \\
\hline 5 & 0.0840 & 0.0085 & 0.00090 & 0.00014 & 0.00007 \\
\hline
\end{tabular}

Table 3 Shear stress for different values of porous parameter $\left(\sigma_{0}\right)$ and Hartmann number $(M)$ at a fixed porous media width $H=h / 4$

\begin{tabular}{|c|c|c|c|c|c|}
\hline$M \downarrow \quad \sigma_{0} \rightarrow$ & $10^{1}$ & $10^{2}$ & $10^{3}$ & $10^{4}$ & $10^{5}$ \\
\hline 0.0 & -8.53340 & 4.5547 & 5.8645 & 5.9942 & 6.0085 \\
\hline 2.0 & -15.0825 & 1.0481 & 2.6617 & 2.8231 & 2.8392 \\
\hline 3.5 & -21.6168 & -0.6726 & 1.4223 & 1.6318 & 1.6527 \\
\hline 5 & -28.2256 & -1.7937 & 0.8498 & 1.1142 & 1.1406 \\
\hline
\end{tabular}

Table 4 Shear stress for different values of porous parameter $\left(\sigma_{0}\right)$ and Hartmann number $(M)$ at a fixed porous media width $H=h / 2$

\begin{tabular}{|c|c|c|c|c|c|}
\hline$M \downarrow \quad \sigma_{0} \rightarrow$ & $10^{1}$ & $10^{2}$ & $10^{3}$ & $10^{4}$ & $10^{5}$ \\
\hline 0.0 & -8.08210 & 4.5970 & 5.8687 & 5.9982 & 6.0086 \\
\hline 2.0 & -14.4858 & 1.0578 & 2.6143 & 2.7700 & 2.7855 \\
\hline 3.5 & -20.6036 & -0.6220 & 1.3778 & 1.5778 & 1.5978 \\
\hline 5 & -26.6069 & -1.6805 & 0.8135 & 1.0629 & 1.0879 \\
\hline
\end{tabular}


K. Ramakrishnan et al. / JAFM, Vol. 6, No. 1, pp. 39-47, 2013.

Table 5 Shear stress for different values of porous parameter $\left(\sigma_{0}\right)$ and Hartmann number $(M)(H=3 h / 4)$

\begin{tabular}{|c|c|c|c|c|c|}
\hline$M \downarrow \quad \sigma_{0} \rightarrow$ & $10^{1}$ & $10^{2}$ & $10^{3}$ & $10^{4}$ & $10^{5}$ \\
\hline 0.0 & -7.6356 & 4.6371 & 5.8726 & 5.9980 & 6.0086 \\
\hline 2.0 & -13.9207 & 1.0653 & 2.5684 & 2.7188 & 2.7338 \\
\hline 3.5 & -19.6722 & -0.6769 & 1.3359 & 1.5272 & 1.5463 \\
\hline 5 & -25.1566 & -1.5803 & 0.7801 & 1.0162 & 1.0398 \\
\hline
\end{tabular}

Table 6 Shear stress for different values of porous parameter $\left(\bar{\alpha} \sigma_{0}\right)$ and Hartmann number $(M)$ in the case of infinite thickness porous media

\begin{tabular}{|c|c|c|c|c|c|}
\hline$M \downarrow \bar{\alpha} \sigma_{0} \rightarrow$ & $10^{1}$ & $10^{2}$ & $10^{3}$ & $10^{4}$ & $10^{5}$ \\
\hline 0.0 & -7.9263 & 3.5949 & 5.0869 & 5.2384 & 5.2577 \\
\hline 2.0 & -13.3746 & 0.4362 & 2.3180 & 2.5130 & 2.5326 \\
\hline 3.5 & -17.6479 & -1.2136 & 1.2166 & 1.4713 & 1.4969 \\
\hline 5 & -21.2751 & -2.3594 & 0.6915 & 1.0154 & 1.0480 \\
\hline
\end{tabular}

\section{CONCLUSION}

The combined effects of porous parameter and Hartmann number on the steady magnetohydro- dynamic flow of a conducting, viscous, incompressible fluid in a uniform channel covered by porous media of finite thickness is studied using BJR slip condition. The result of the present work shows that the axial velocity of the fluid is reduced by porous parameter and Hartmann number. As expected from physical consideration, it was observed that the shear stress was small for small values of the porous parameter and it exhibited an increasing trend with increasing porous parameter. Further, an elaborate comparison of the results obtained in the present work using the BJR slip condition has been provided with those of our earlier work using the BJ slip condition.

\section{REFERENCES}

Beavers, G.S. and Joseph, D.D., (1967), Boundary conditions at a naturally permeable wall, J. Fluid Mech. 30, 197-207.

Burton, A.C., (1966), Physiology and Biophysics of the Circulation, Introductory Text, Year Book Publisher, Chicago.

Chandrasekhar, S.(1961). Hydrodynamic and Hydro magnetic Stability, Oxford University Press London.

Chaturani, P. and Kaloni, P.N., (1976), Two-layered poiseuille flow model for blood flow through arteries of small diameter and arterioles, Biorheology, 13, 243-250.

Chaturani, P. and Upadhya, V.S., (1979), A two-layered model for blood flow through small diameter tubes, Biorheology, 16, 109-118.

Chaturani, P. and Biswas, D., (1983), A theoretical study of blood flow through stenosed arteries with velocity slip at the wall, Proc. First Internat. Symposium on Physiological Fluid Dynamics, IIT Madras, India, 23-26.

Chandrasekhara, B.D. and Rudraiah, N., (1980), MHD flow through a channel of varying gap, Indian. J. Pure and Appl. Math. 11(8), 1105-1123.

Copley, A.L., (1990). Fluid mechanics and rheology, Biorheology. 27, 3-19.

Darcy, H., (1856). Les fountains Publique De La Ville De Dijon, Delmont, Paris.

Haik, Y.,Pai, V. and Chen, C.J., (1999). Development of magnetic device for cell separation, J. Magn. Magn. Mater., 194, 261.

Higashi, T., Yamagishi, A., Takeuchi, T., Kawaguchi, N., Sagawa, S., Onishi, S. and Date, M., (1993). Orientation of Erythrocytes in a strong static magnetic field, J. Blood . 82(4), 1328-1334.

MacDonald, D.A. (1974), Blood flow in arteries, 2nd ed., London: Edward Arnold Publishers.

Majhi, S.N. and Usha, L., (1984), Fahraeus-Lindqvist effect and generalized poiseuille flow with or without wall slip, Proc. $13^{\text {th }}$ Nat. Conf. Fluid Mech. Fluid Power, REC, Thiruchirappalli, India, 125-128.

Makinde, O.D. and Osalusi, E. (2006), MHD flow in a channel with slip at the permeable boundaries, Rom. Journ. Phys., 51(3-4), 319-328.

Misra, J.C. and Chakravarty, S. (1986), Flow in arteries in the presence of stenosis, J. Biomech., 19, 907-918.

Misra, J.C. and Shit, G.C. (2007), Effect of Magnetic field on blood flow through an artery: A numerical model, Tom., 12(4), 3-16.

Ng, C.O., Rudraiah, N. and Nagaraj, C. (2008), Electrohydrodynamic pulsatile flow in a channel bounded by porous layer of smart material, Int. J. Engg. Sci., 5, 1-13.

Pal, D., Veerabhadraiah, R., Shivakumar, P.N. and Rudraiah, N., (1984), Longitudinal dispersion of tracer particles in a channel bounded by porous 
K. Ramakrishnan et al. / JAFM, Vol. 6, No. 1, pp. 39-47, 2013.

media using slip condition, Int. J. Math. Math.Sci., 7, 755-764.

Philip, D. and Peeyush Chandra (1996), Flow of eringen fluid(simple micro fluid) through an artery with mild stenosis, Int. J. Eng. Sci., 34, 87-99.

Ramakrishnan, K. and Shailendhra, K. (2011), Hydromagnetic flow through uniform channel bounded by porous media, Appl. Math. Mech.-Engl. Ed., 32(7), 837-846.

Roberts, P.H. An introduction to magneto- hydrodynamics, Longmans publications, London, 1967.

Rudraiah, N. (1962), Magnetohydrodynamic stability of heterogeneous incompressible non-dissipative conducting liquids, Appl. Sci. Res., 11, 105-117.

Rudraiah, N. (1985), Coupled parallel flows in a channel and a bounding porous medium of finite thickness, Trans. ASME . 107(3), 322-329.

Rudraiah, N., Chiu-on Ng, Nagaraj, C. and Nagaraj, H.N., (2006), Electrohydrodynamic dispersion of Macromolecular components in biological bearing, Journal of Energy, Heat and Mass transfer, 28, 261-280.
Rudraiah, N., Chiu-on Ng and Nagaraj, C., (2007), Electrohydrodynamic dispersion of Macromolecular components in biological bearing Effect of Artificial cartilage made up of smart material of nanostructure on dispersion in poorly conducting macromolecular components in synovial joints, Key Engineering Materials, 334-335, 1245-1248.

Ruuge, E.K. and Rusetski, A.N. (1993), Magnetic fluid as drug carriers : Targeted transport of drugs by a magnetic field,, J. Magn.Magn.Mater, 122, 335.

Shivakumar, P.N., Nagaraj, S., Veerabhadraiah, R. and Rudraiah, N. (1986), Fluid Movement in a channel of varying gap with permeable walls covered by porous media, Int. J. Engng. Sci., 24(4), 479-492.

Shukla, J.B., Parihar, R.S. and Gupta, S.P. (1980), Effects of peripheral layer viscosity on blood flow through the artery with mild stenosis, Bull.Math. Biol., 42, 797-805.

Tzirtzilakis, E.E., (2005), A Mathematical model for blood flow in a magnetic field, Physics of Fluids 